Original Paper http://ajol.info/index.php/ijbcs http://indexmedicus.afro.who.int

\title{
Prévention du brunissement lors de l'extraction de jus de banane plantain et valorisation des résidus issus de cette extraction
}

\author{
Mohamed Anderson $\mathrm{YEO}^{1 *}$, Ernest Kouadio KOFFI ${ }^{1}$, \\ Olivier Kouamé CHATIGRE ${ }^{1}$ et Eric Fattoh ELLEINGAND ${ }^{2}$
}

\author{
${ }^{I}$ Laboratoire de Biochimie Sciences des Aliments, UFR Biosciences, Université Félix Houphouët \\ Boigny de Cocody, UFHB 01 BP V 34 Abidjan, Côte d'Ivoire. \\ ${ }^{2}$ Laboratoire de Génie Chimie et Agroalimentaire, Institut Nationale Polytechnique Félix \\ Houphouët Boigny INP/HB, 1093 Yamoussoukro, Côte d'Ivoire. \\ *Corresponding author; E-mail : ymoander@yahoo.fr; 22 bp 815 abidjan 22 ; Tel : (225)09 124208
}

\section{REMERCIEMENTS}

Cette recherche a été soutenue financièrement par le fond PPAAO (Programme de Productivité Agricole en Afrique de l'Ouest) pour la Banque mondiale.

\section{RESUME}

Ces expériences ont été conduites dans l'optique d'évaluer d'une part la capacité de divers traitements anti brunissement à prévenir le brunissement du jus de banane plantain et d'autre part de valoriser les résidus issus de cette extraction sous forme de confiture. Le métabisulfite de potassium (100 $\mathrm{mg} / \mathrm{kg}$ ) était plus efficace pour la production d'un jus de banane légèrement coloré et de coloration stable par rapport aux deux méthodes de traitement thermique (chauffage de la banane entière non épluchée à $100{ }^{\circ} \mathrm{C}$ pendant 11 min et le chauffage de la purée à $85^{\circ} \mathrm{C}$ pendant $1-2 \mathrm{~min}$ et l'acide ascorbique $(470 \mathrm{mg} / \mathrm{kg})$. La polyphenoloxydase était plus active dans les jus clarifiés traités à l'acide ascorbique et le témoin qui brunissent tous au fil du temps. L'analyse sensorielle a révélé que tous les jus présentaient la même intensité de flaveur banane plantain. Les résidus d'extraction des jus ont été valorisés par la production de deux types de confiture (avec ou sans ajout de poudre de baobab comme source de pectine) appréciés par les dégustateurs. Par ailleurs, la confiture contenant la poudre de baobab (Adansonia digitata) aura une meilleure conservation grâce à son $\mathrm{pH}$ plus acide.

(C) 2015 International Formulae Group. All rights reserved.

Mots clés : Plantain, brunissement, extraction, valorisation, jus, Adansonia digitata.

\section{Prevention of browining in the preparation of clarified plantain juice and valorization of processing waste}

\begin{abstract}
These experiments were conducted with a view to evaluating both the ability of various anti browning treatments to prevent browning of plantain juice and also to value the residues from the extraction as jam. Potassium metabisulfite $(100 \mathrm{~m} / \mathrm{L})$ was more effective for the production of a slightly colored banana juice
\end{abstract}


with stable color compared to the two methods of thermal treatment (heating the whole banana unpeeled at 100 ${ }^{\circ} \mathrm{C}$ for $11 \mathrm{~min}$ and heating the mash at $85{ }^{\circ} \mathrm{C}$ for $1-2 \mathrm{~min}$ ) and ascorbic acid $(470 \mathrm{mg} / \mathrm{L})$. The polyphenol oxidase was more active in the clarified juice treated with ascorbic acid and the control which darkened over time. Sensory analysis revealed that all the juices had the same intensity of plantain flavor. The juice processing wastes were valued by the production of two types of jam (with or without addition of baobab powder as a source of pectin) appreciated by the tasters. Moreover, jam containing baobab (A digitata) powder will have better preservation with its more acidic $\mathrm{pH}$.

(c) 2015 International Formulae Group. All rights reserved.

Keywords : Plantain, browning, extraction, valorization, juice, Adansonia digitata.

\section{INTRODUCTION}

En Côte d'Ivoire, le plantain a toujours été un aliment de base traditionnel très important pour les populations rurales et citadines; ce qui lui a valu la $2^{\text {ème }}$ place au niveau des denrées consommées après le riz (Soler et N'da, 1990). Selon les statistiques agricoles; (ANADER, 1998; MINAGRI, 2004), la production de banane plantain augmente de façon linéaire. Entre 1990 et 1995, le tonnage de banane plantain s'est amélioré de 4,2\% en moyenne par an (Adopo et al., 1998) pour atteindre 1.300 .000 tonnes en 1997, sur une superficie totale de 216.667 hectares.

Le secteur plantain est encore traditionnel et informel. En effet, ni la production, ni la commercialisation, ni la distribution ne sont organisées. La plupart des pistes de desserte des plantations sont en mauvais état. En période de pluie, une bonne partie de la production est abandonnée sur les lieux de production.

Ainsi $40 \%$ de la production de banane plantain sont perdus en milieu rural. Ces pertes sont aussi dues au manque d'infrastructures routières pour l'acheminement vers les zones urbaines de consommation.

Au niveau mondial, un faible pourcentage de la production bananière est transformée en purée (Stover et Smmonds, 1987 ; Palmer, 1971). Etant donné la teneur élevée en sucres et la flaveur banane universellement appréciée, la production de jus de banane plantain clarifié et stabilisé contribuera à réduire non seulement les pertes post-récoltes mais aussi à proposer un nouveau produit.

$\mathrm{Au}$ nombre des problèmes rencontrés lors de la production du jus de banane, figurent la viscosité élevée de la purée, les difficultés d'extraction du jus (Dupaigne et Dalnic, 1965 ; Viquez et al., 1981) ainsi que le brunissement (Galeazzi et Sgarbieri, 1981). Des études préliminaires sur la production de jus de banane douce ont utilisé diverses méthodes d'extraction et de clarification du jus (Dalnic 1965 ; Dupaigne, 1974 ; Casimir et Jayarama, 1971 ; Sreekantlah et al., 1971 ; Anon, 1981 ; Dupaigne et al., 1971 ; Viquez et al., 1981 ; Koffi et al., 1991). Les méthodes prometteuses sont celles qui utilisent la pectinase et la centrifugation, (Dupaigne, 1974; Viquez et al., 1981; ByaruagabaBaziraka et al., 2012). Néanmoins, des recherches additionnelles sont nécessaires pour évaluer la capacité des pectinases à extraire le jus de la pulpe de banane plantain.

Cette difficulté à extraire le jus de banane, réside essentiellement en sa composition.

En effet, les bananes mûres pourraient encore contenir 1 à $4 \%$ d'amidon (United Fruit CO., 1961) et de la pectine, qui ont un impact sur l'extraction du jus et sa qualité.

En outre, la dopamine, un composé majeur de la banane, entraîne le brunissement de son jus.

Les travaux de Koffi et al. (1991) sur la banane douce ont su indiquer que l'usage de la pectinase associé à diverses méthodes physiques et chimiques permet de clarifier le jus et de préserver sa couleur. 
L'objectif de cette étude est d'évaluer l'effet des méthodes thermiques et chimiques sur le brunissement du jus de banane plantain et la valorisation des résidus d'extraction par la production de confitures.

\section{MATERIEL ET METHODES Matériel biologique}

Le matériel végétal était essentiellement constitué de la banane plantain mûre (Musa paradisiaca) au stade 8 (couleur jaune avec des tâches noires) pour le jus et des résidus d'extraction supplémentés ou non de poudre de baobab pour la production de confitures.

\section{Méthode de prévention du brunissement}

Diverses méthodes communément utilisées nous ont permis de mener des tests dans la prévention du brunissement lors de la production du jus de banane plantain clarifié.

La première méthode a consisté à chauffer la banane entière, non épluchée pendant 11 minutes à la vapeur $\left(100^{\circ} \mathrm{C}\right)$. Des recherches antérieures ont montré que ce traitement thermique était adéquat pour l'inhibition de la polyphenoloxydase de la banane douce (Koffi et al., 1991) (Figure 1). Après chauffage, la banane est épluchée à l'aide d'un couteau stérile en acier inoxydable.

La pulpe a été broyée et la purée obtenue a été acidifiée jusqu'à $\mathrm{pH}$ 4,0 en utilisant de l'acide citrique $(20 \%)$ pour produire un aliment acide. La purée est ensuite ramenée à la température ambiante et on ajoute la pectinase (1 g/200ml d'eau distillée) (Ezoua, 1995), et l'ensemble est incubé pendant 2 heures à $25^{\circ} \mathrm{C}$.

Une autre méthode de traitement thermique a consisté à chauffer la purée de banane plantain dans une couscoussière jusqu'à $85^{\circ} \mathrm{C}$ pendant 1 à $2 \mathrm{mn}$ (Koffi et al., 1991) (Figure 2). La purée a été refroidie rapidement à $25^{\circ} \mathrm{C}$ dans un bac d'eau glacée, acidifiée jusqu'a $\mathrm{pH}=4,0$ avec l'acide citrique $(20 \%)$, et incubée pendant 2 heures après ajout de la solution de pectinase. Deux autres traitements ont consisté en l'incorporation de métabisulfite de sodium (100 mg/kg) ou d'acide ascorbique (470 $\mathrm{mg} / \mathrm{kg}$ ) à la purée immédiatement après le broyage de la pulpe de banane (Koffi et al., 1991 ; Galeazzi et Sgarbierl, 1981) (Figure 3).

Les purées ont été acidifiées jusqu'à pH 4,0 et incubées après ajout de la solution de pectinase pendant 2 heures. Un témoin n'ayant subi aucun traitement thermique ni ajout d'additifs a été acidifié et traité avec la solution de pectinase dans les mêmes conditions. Tous ces essais ont été effectués en triple.

A la suite du traitement enzymatique, la purée homogénéisée subit une centrifugation à l'aide d'une centrifugeuse de marque Bofuge III. Le jus de banane plantain est donc obtenu après centrifugation à 3000 tours/min pendant $15 \mathrm{~min}$. Le jus est par la suite filtré avec un tamis possédant des mailles de $0,250 \mathrm{~mm}$ de diamètre; puis 200 $\mathrm{mg} / \mathrm{kg}$ d'acide benzoïque ont été ajoutés afin de le préserver de la prolifération microbienne.

L'embouteillage s'est fait dans des bouteilles pré stérilisées de $250 \mathrm{ml}$.

Le degré de brunissement a été évalué par la mesure de l'absorbance. (Abs) à $420 \mathrm{~nm}$ au spectrophotomètre.

\section{Evaluation de la stabilité}

Pour l'évaluation de la stabilité de la couleur du jus, $250 \mathrm{mg} / \mathrm{kg}$ d'acide benzoïque supplémentaires ont été ajoutés à $100 \mathrm{ml}$ d'échantillon de jus et recouvert avec un para film et l'absorbance a été mesurée de nouveau après $48 \mathrm{~h}$ comme décrit précédemment.

\section{Mesure de l'activité de la polyphénoloxydase}

L'activité de la Polyphénol oxydase (PPO) a été déterminée en mesurant le taux d'accroissement de l'absorbance à $420 \mathrm{~nm}$ et à $25{ }^{\circ} \mathrm{C}$ à l'aide d'un spectrophotomètre de marque PERKIN-ELMER Lamda 1A uv/vis. Le milieu d'incubation contenait $1 \mathrm{ml}$ de catéchol $(0,5 \mathrm{M}), 1,9 \mathrm{ml}$ de tampon sodium phosphate (monobasique $0,05 \mathrm{M}$ ) à $\mathrm{pH} 6,5$ et $1 \mathrm{ml}$ de jus de banane plantain. L'activité de la polyphenoloxydase est exprimée en unité de changement de l'absorbance par minute par $\mathrm{ml}$ à $25^{\circ} \mathrm{C}$. 


\section{Production de la confiture}

Les résidus issus de l'extraction du jus de banane plantain mûre, sont transformés en confiture conformément aux exigences de la FAO (1997).

Une première confiture sera produite sans ajout de poudre de baobab et la seconde, en utilisant la poudre de baobab comme source de pectine (Figure 4).

En utilisant les résultats de travaux antérieurs de Ndabikunze et al. (2011), $50 \mathrm{~g}$ de poudre de baobab sont additionnés à 1 Litre de purée de banane plantain en lieu et place de la pectine commerciale.

La quantité de sucre à ajouter est déterminée par la formule suivante :

Sucre à ajouter $=\frac{[\text { TSS (final) })-\text { TSSS(résidus })]^{\prime}}{100} \mathrm{xW}$

TSS $($ final $)=$ Extrait sec soluble, niveau normal requis de la confiture (69\%)

TSS (résidus) $=$ Extrait sec soluble des résidus

$\mathrm{W}=$ Poids en gramme des résidus à utiliser

Le mélange de sucre et de résidus est mis à cuire jusqu'à obtention d'un extrait sec soluble de $69 \%$ mesuré à l'aide d'un réfractomètre de poche. .

La confiture ainsi obtenue est renversée dans un bocal en verre stérile, et retournée après fermeture afin de stériliser le couvercle du bocal.

\section{Analyse statistique et sensorielle}

Les jus ont été évalués par un panel de 25 personnes expérimentées. Chaque paneliste a évalué les jus par rapport à l'intensité de la flaveur banane plantain.

A chaque jus a été attribué une note sur l'échelle graduée de 1 à 9 , avec 1 correspondant respectivement à une très faible intensité de flaveur et 9 une très forte intensité de flaveur banane plantain. Des verres sombres ont été utilisés afin de masquer toute différence de couleur au niveau des échantillons. Toutes les valeurs mesurées ont été soumises à l'analyse de variance avec le logiciel Graphad 5.01. Les comparaisons entre les variables dépendantes ont été déterminées par analyse de variance ANOVA, test de Duncan, test de Newman Keuls, analyse de corrélation. La signification statistique a été définie au niveau $5 \%$ et le profil a été réalisé au logiciel TASTEL+.

\section{RESULTATS}

Prévention du brunissement

La prévention du brunissement par chauffage de la purée à $85^{\circ} \mathrm{C}$ s'avère être la plus efficace des méthodes $(0,022)$ suivie de celle des sulfites $(0,047)$. Le jus obtenu après chauffage de la purée à $85^{\circ} \mathrm{C}$ est moins brun (coloré) comme l'indique l'absorbance à 420 $\mathrm{nm}$ (Tableau 1). Les jus extraits après traitement de la purée à $85{ }^{\circ} \mathrm{C}$, au méta bisulfite et à l'acide ascorbique (Tableau 1), présentent une absorbance plus faible que celle du témoin. C'est donc la preuve que ces traitements ont une efficacité relative dans la prévention du brunissement.

\section{Evaluation de la stabilité}

Après 48 heures d'exposition à la température ambiante, le jus obtenu par traitement au méta bisulfite à raison de 100 $\mathrm{mg} / \mathrm{kg}$ ou par chauffage à $85^{\circ} \mathrm{C}$ de la purée de banane, ont une faible absorbance, respectivement $(0,334$ et 0,275$)$, donc un brunissement plus faible que le jus obtenu après traitement de la banane entière à $100^{\circ} \mathrm{C}$, $(0,765)$, suivi du jus extrait de la banane traitée à l'acide ascorbique $(0,742)$ (Tableau $1)$.

\section{Mesure de l'activité de la polyphénoloxydase \\ La polyphénoloxydase ( $\mathrm{PPO}$ ) a été très} active dans le jus traité à l'acide ascorbique $(470 \mathrm{mg} / \mathrm{kg}$ ) et le jus témoin, mais pratiquement inactive sinon très peu dans les jus issus des traitements thermiques (banane entière non épluchée chauffée à $100{ }^{\circ} \mathrm{C}$ et la purée chauffée à $85^{\circ} \mathrm{C}$ ) et celui traité par le metabisulfite de sodium.

\section{Caractéristiques physico-chimiques de la confiture}

Les résultats du Tableau 2 rendent compte de la composition physico-chimique de la pulpe de banane plantain, de la poudre de baobab (Adansonia digitata L.) et des confitures C500 et C100 respectivement 
(confiture des résidus d'extraction du jus de pulpe de banane plantain mûre (stade 8) avec supplément de poudre de baobab à $50 \mathrm{~g} / \mathrm{L}$ de pulpe et sans poudre de baobab.

Le taux d'extrait sec réfractométrique est significativement très élevé au niveau des résidus d'extraction du jus $(\mathrm{p}<0,05)$ tandis que le taux le plus bas est contenu dans la poudre de baobab.

L'acidité titrable (TTA) est plus élevée dans la poudre de baobab $(0,51)$ et faible dans les résidus de banane plantain (Tableau 2).

\section{Analyse microbiologique}

L'analyse de la flore totale des différentes confitures résumées dans le
Tableau 3 montre une qualité satisfaisante. Ces confitures présentent des valeurs de flore totale inférieures à $10^{-2} \pm 0,03 \mathrm{ufc} / \mathrm{g}$ pour la confiture sans poudre de pulpe de baobab et à $10^{-2} \pm 0,03 \mathrm{ufc} / \mathrm{g}$ pour la confiture contenant $50 \mathrm{~g} / \mathrm{L}$ de poudre de pulpe de baobab.

\section{Analyse sensorielle}

La Figure 5 laisse apparaitre des caractéristiques sensorielles moyennes des confitures statistiquement différentes. Les caractéristiques étudiées sont: la couleur, la malléabilité, le goût sucré, l'acidité et l'appréciation générale des confitures.

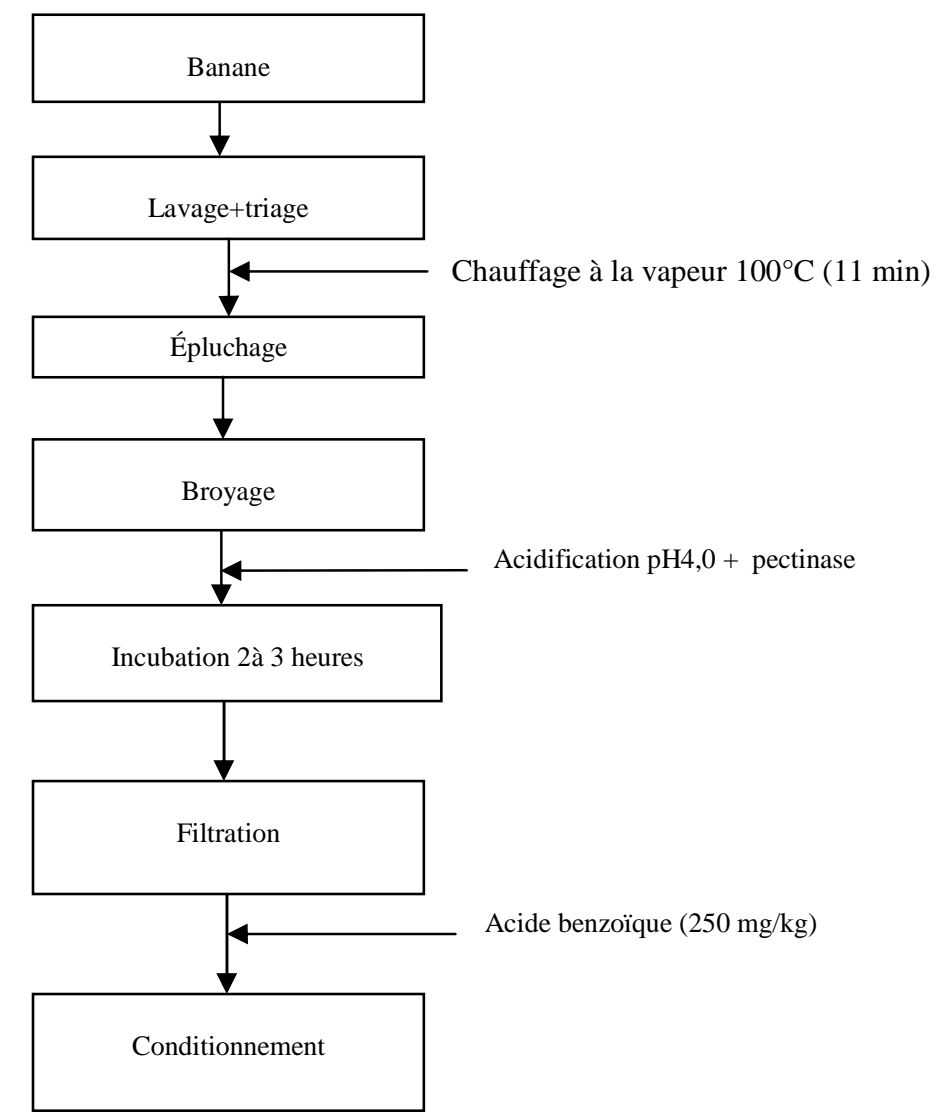

Figure 1: Prévention du brunissement du jus par chauffage de la banane entière non épluchée. (Koffi et al., 1991, modifié). 


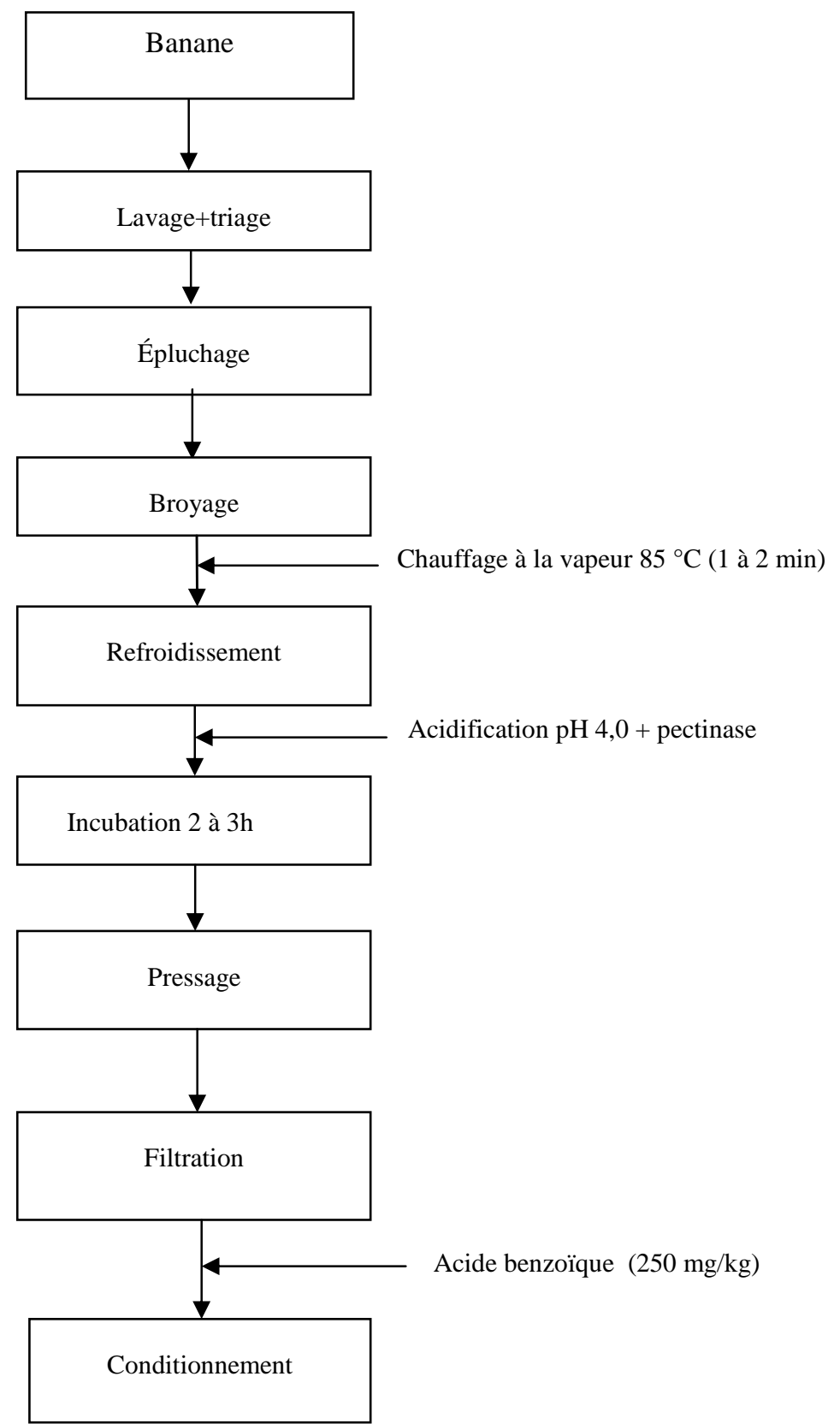

Figure 2: Prévention du brunissement du jus par chauffage de la purée de banane à $85{ }^{\circ} \mathrm{C}$. (Koffi et al., 1991, modifié). 


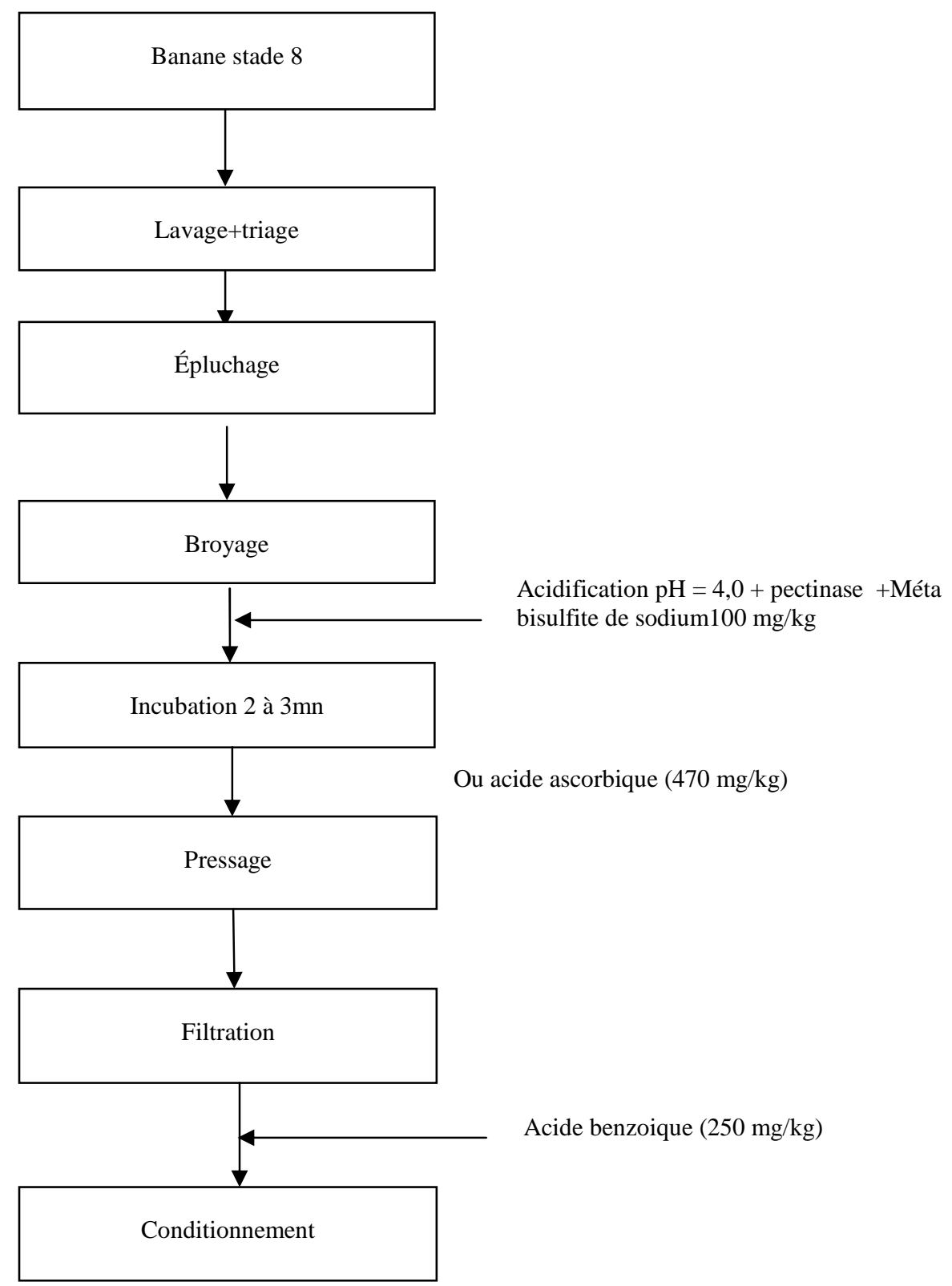

Figure 3 : Prévention du brunissement par utilisation du métabisulfite de sodium ou de l'acide ascorbique. (Koffi et al., 1991, modifié). 


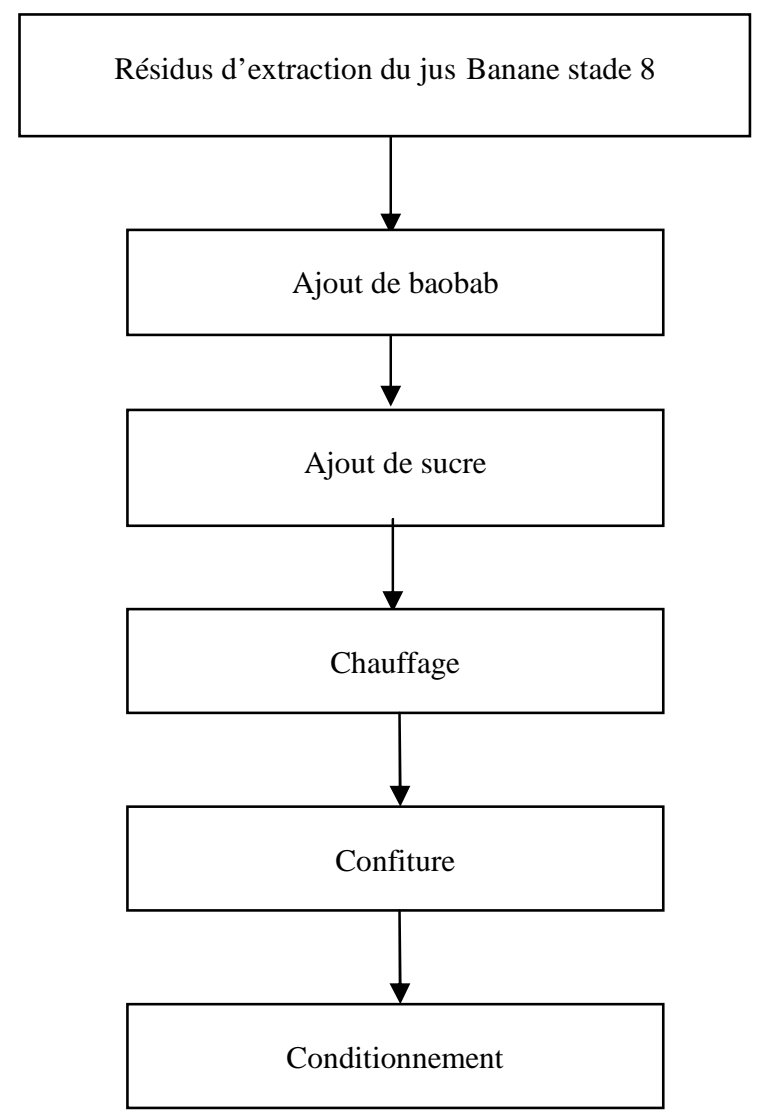

Figure 4 : Diagramme de production de la confiture à partir des résidus d'extraction du jus de pulpe de banane plantain mure (stade 8 ).

Tableau 1 :Effet de la chaleur, du métabisulfite, de l'acide ascorbique sur l'absorbance et évaluation sensorielle du jus clarifié de banane plantain (stade 8).

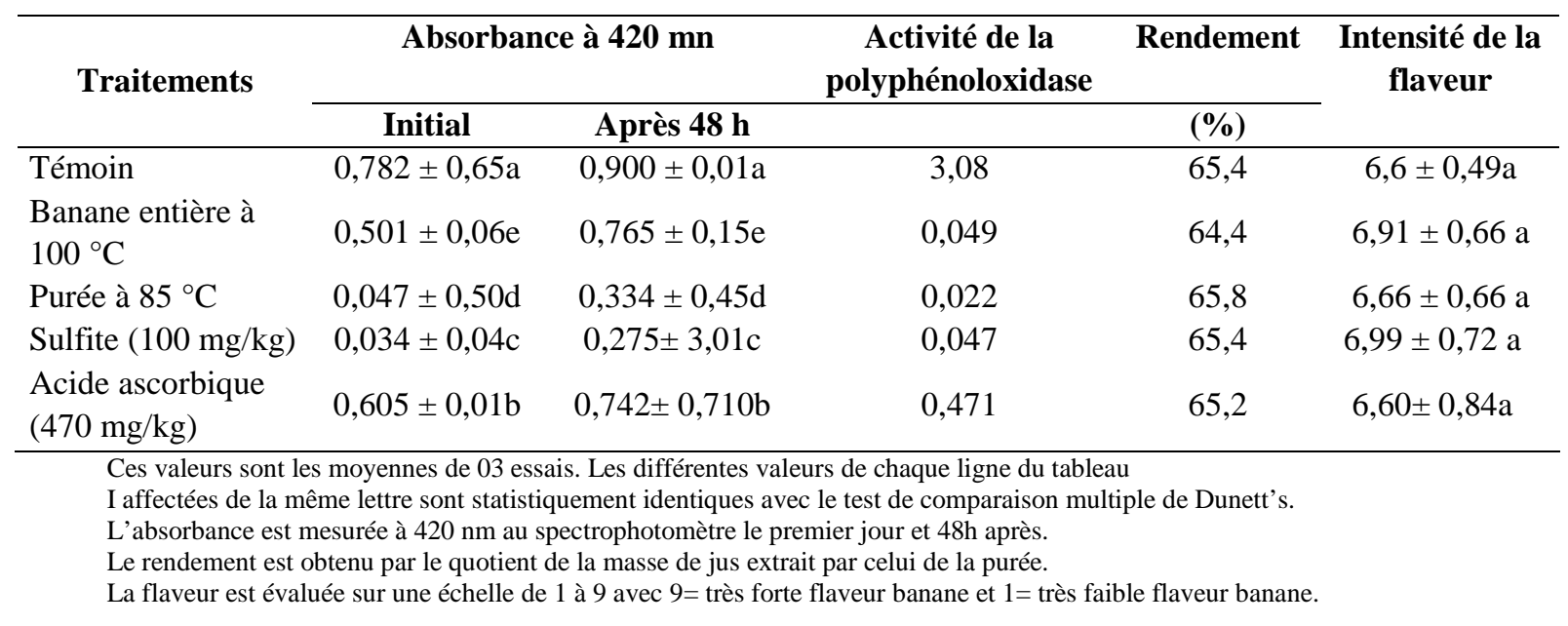


M. A. YEO et al. /Int. J. Biol. Chem. Sci. 9(4): 1809-1821, 2015

Tableau 2: Paramètres physico-chimiques des résidus d'extraction des jus, de la poudre de Adansonia digitata L.et des confitures à 100\% de résidus de banane plantain et $50 \mathrm{~g} / \mathrm{l}$ de poudre de baobab.

\begin{tabular}{|c|c|c|c|c|c|}
\hline \multirow[t]{2}{*}{ Echantillons } & \multirow[t]{2}{*}{ pH } & $\begin{array}{c}\text { Extrait sec } \\
\text { soluble } \\
(\text { obrix })\end{array}$ & \multirow[t]{2}{*}{$\begin{array}{c}\text { Acidité } \\
\text { titrable } \\
\text { (méq.g/l) }\end{array}$} & Matière sèche & Humidité \\
\hline & & (\%brix) & & $(\%)$ & $(\%)$ \\
\hline Résidus d'extraction du jus & $5,08 \pm 0,00^{b}$ & $21,97 \pm 0,07^{b}$ & $0,51 \pm 0,01^{d}$ & $31,19 \pm 0,02^{\mathrm{c}}$ & $68,81 \pm 0,02^{b}$ \\
\hline Adansonia digitata $\mathrm{L}$. & $3,37 \pm 0,03^{d}$ & $11,64 \pm 0,02^{\mathrm{c}}$ & $2,26 \pm 0,02^{a}$ & $83,12 \pm 0,02^{\mathrm{a}}$ & $16,89 \pm 0,03^{\mathrm{d}}$ \\
\hline Confiture à $100 \%$ de résidus de banane plantain & $5,32 \pm 0,02^{a}$ & $69 \pm 0,00^{\mathrm{a}}$ & $1,51 \pm 0,25^{\mathrm{c}}$ & $32,33 \pm 0,02 \mathrm{~b}$ & $67,67 \pm 0,02^{c}$ \\
\hline Confiture avec $50 \mathrm{~g} / \mathrm{l}$ de poudre de baobab & $4,67 \pm 0,01$ & $69 \pm 0,00^{\mathrm{a}}$ & $2,06 \pm 0,05^{\mathrm{b}}$ & $27,84 \pm 0,01^{\mathrm{d}}$ & $72,16 \pm 0,01^{a}$ \\
\hline
\end{tabular}

Les valeurs de la même colonne suivies de la même lettre ne sont pas statistiquement différentes à p<0,05 (n=3).

Tableau 3: Flore totale (ufc/g) des confitures de résidus d'extraction du jus de pulpe de banane plantain mure (stade 8$)(\mathrm{n}=3)$

\begin{tabular}{lc}
\hline Confitures & Flore totale $($ ufc/g) \\
\hline Confiture à $100 \%$ de résidus de banane plantain & $<1 \times 10-2 \pm 0,03 \mathrm{a}$ \\
Confiture avec $50 \mathrm{~g} / \mathrm{l}$ de poudre de baobab & $<1 \times 10-2 \pm 0,03 \mathrm{~b}$ \\
\hline \multicolumn{1}{c}{ Les coefficients affectés de lettres différentes sont significativement différents. $(\mathrm{P}<0,05) ; \mathrm{n}=3}$.
\end{tabular}




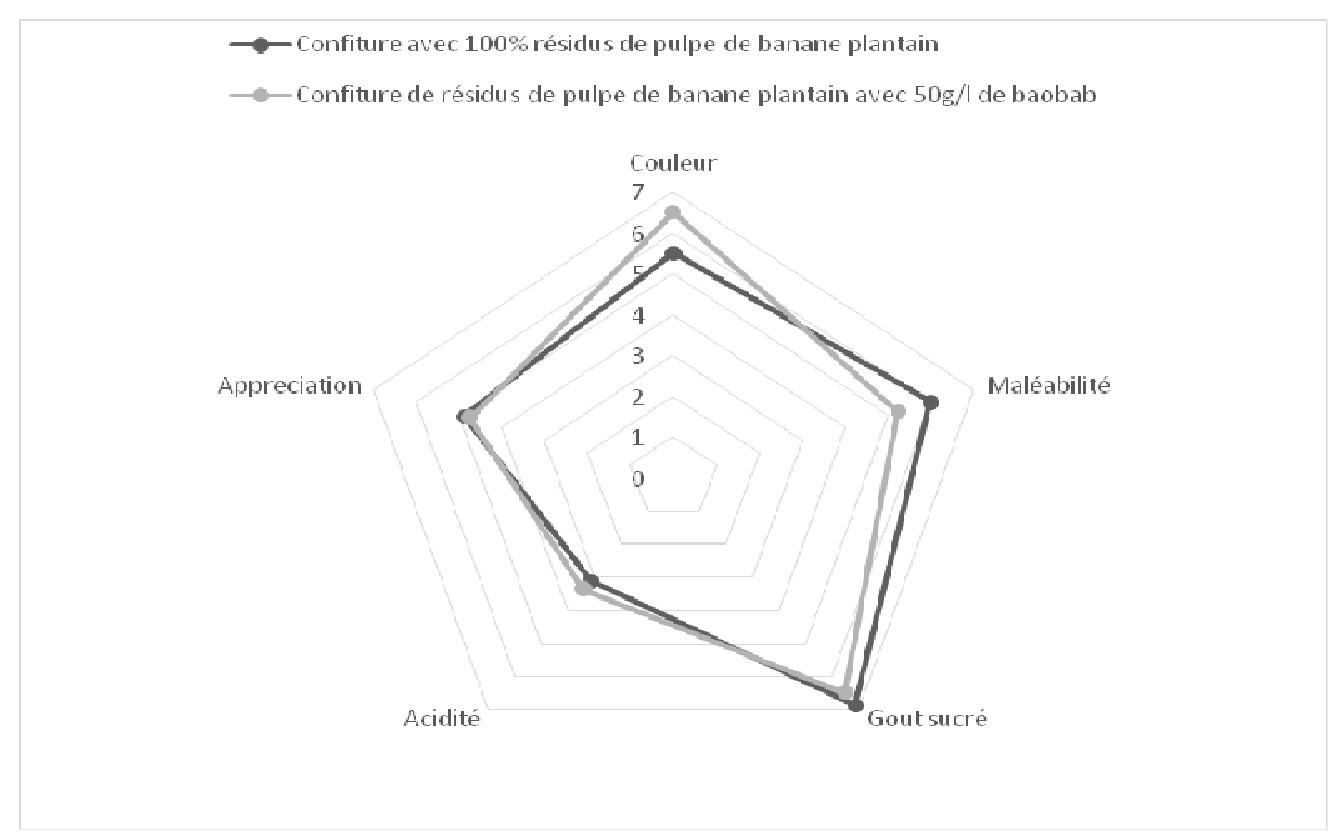

Figure 5 : Profil sensoriel des confitures avec $50 \mathrm{~g} / \mathrm{L}$ de baobab et sans baobab.

\section{DISCUSSION}

Les températures appliquées lors du blanchiment varient considérablement en fonction du végétal à traiter et des enzymes à inactiver. En général, une exposition des PPO à des températures de l'ordre de $70-90{ }^{\circ} \mathrm{C}$ entraîne la destruction de leur activité catalytique (Vámos-Vigyázó, 1981). La purée traitée à l'acide ascorbique brunit considérablement quand elle est exposée à l'air. Les antioxygènes sont des composés capables de retarder l'oxydation par des mécanismes indirects tels que la complexation des ions métalliques ou la réduction de l'oxygène. Parmi ceux-ci, l'acide ascorbique (vitamine C) a la propriété d'être fortement réductrice. Cette molécule agit sur l'oxygène par oxydoréduction grâce à sa fonction ènediol et se transforme en acide déhydroascorbique qui a la même activité biologique que l'acide ascorbique (Jehl et al., 2004).

Les résultats obtenus à la suite de la prévention du brunissement sont comparables à ceux de Koffi et al., (1991) dans une étude similaire réalisée avec la banane dessert.
En effet, ces résultats confirment que le métabisulfite présente une efficacité dans la prévention des brunissements au niveau de la banane en général. D'après Madero et Finne (1982), les sulfites ont un effet inhibiteur compétitif sur la PPO en se liant à un groupement sulfhydryl du site actif. Par ailleurs, Ferrer et al. (1989) suggèrent que l'inhibition du brunissement enzymatique par les sulfites est due à la réaction des sulfites sur les quinones, formant ainsi des sulfoquinones, inhibiteurs irréversibles de la PPO et provoquant ainsi son inactivation.

Les résultats consignés dans le Tableau 1 résument l'intensité de la flaveur des différents jus. Il n'y a pas de différence significative au niveau de l'intensité de la flaveur banane des jus obtenus après les différents traitements appliqués à la banane plantain.

Les jus présentent tous une flaveur audessus de la moyenne avec toutefois une légère préférence pour le jus obtenu après traitement de la purée de banane au méta bisulfite $(100 \mathrm{mg} / \mathrm{kg})$. 
Selon Embs et Markakis (1965), ces sulfites inhibent non seulement la PPO mais atténuent le brunissement par combinaison avec les O-quinones, prévenant la formation des mélanines par condensation. Le chauffage de la banane entière non épluchée a été efficace pour l'inactivation de la PPO. Ce résultat est en accord avec ceux de plusieurs chercheurs (Mao 1974; Koffi et al., 1991) mais ce traitement thermique aurait une efficacité réduite due au fait du brunissement non-enzymatique occasionné par la migration de composés oxydables (tels que les composés phénoliques) provenant de la peau de banane vers la pulpe lors du chauffage (Koffi et al., 1991).

Le rendement de l'extraction du jus de banane plantain clarifié obtenu est en moyenne de $65 \%$ (Tableau 1), ce qui représente une baisse de $10 \%$ par rapport à celui du jus de banane dessert de Koffi et al. (1991) mais identique à celui de Viquez et al. (1981).

L'analyse de la flore totale des différentes confitures prouve que l'embouteillage dans des bocaux précédemment préstérilisés et leur remplissage à chaud avec retournement s'est avéré efficace et adéquate pour la prévention du développement microbien.

L'efficacité de ces méthodes thermiques et du sulfitage a été également prouvée par Koffi et al. (1991) en inactivant totalement la PPO dans le jus de banane dessert par les mêmes procédés (thermique et le sulfitage).

La valeur de l'acidité titrable de la poudre de A. digitata est comparable à celle obtenue par Belitz et Grosch (1999), dans la production de confiture des fruits conventionnels.

Le niveau de l'acidité dans les fruits a une influence sur la gélification; important aspect dans la production de la confiture. En effet, l'acidité totale titrable affecte la cinétique de réaction et donc la consommation en calcium. En fonction du milieu et par exemple des fruits utilisés, l'acidité totale guidera, comme le pH, les quantités nécessaires de calcium à ajouter. Pour des teneurs en acide élevées, les gels sont plus fermes car ils lient mieux le calcium et en plus grande quantité. Il se produit des changements physiques ou chimiques qui tendent à diminuer la solubilité de la pectine, favorisant la formation de cristallisations locales (Eric, 1990).

En outre, le $\mathrm{pH}$ de la confiture de résidus du jus de banane plantain est significativement élevé $\quad(p<0,05)$ en comparaison avec celui atteint lors de la production de la confiture des pulpes des fruits transformés (Ndabikunze et al., 2011). L'ajout de poudre de baobab s'avère indispensable pour acidifier les résidus tout en améliorant la teneur en pectine.

Globalement plus appréciée que la confiture avec $50 \mathrm{~g} / \mathrm{L}$ de poudre de pulpe de baobab $(4,75 \pm 1,10)$, la confiture de résidus de pulpe de banane plantain sans baobab, est moins acide $(3,125 \pm 1,36)$ mais plus sucrée $(6,875 \pm 1,51)$.

Les confitures produites à partir des résidus issus de l'extraction du jus de pulpe de banane plantain mûre (stade 8) donnent des valeurs d'extrait sec réfractométrique et d'acidité titrable, comparables à certaines valeurs conventionnelles, telles que celles des mangues $(14 \%)$, requises pour la production de confitures (Belitz et al., 1999).

L'apport de poudre de baobab (Adansonia digitata L.) a permis de réaliser la balance de l'équilibre entre le $\mathrm{pH}$ des pulpes, la quantité de sucre à ajouter et la teneur en pectine afin d'obtenir une confiture à texture appréciable telle que décrite par Belitz et al. (1999).

\section{Conclusion}

Les résultats de ces expériences nous montrent que même si les traitements thermiques présentent une efficacité relative à prévenir le brunissement du jus, le métabisulfite reste la méthode la plus efficace.

Avec des flaveurs statistiquement identiques, l'on constate une légère préférence 
pour le jus extrait de la purée traitée au métabisulfite. La production de la confiture à partir des résidus issus de l'extraction de jus de pulpe de banane plantain mûre (stade 8) permet effectivement de valoriser ces résidus.

Du point de vue sensoriel la, poudre de baobab n'altère en rien l'appréciation des dégustateurs pour cette confiture. Ce fruit pourrait donc constituer une alternative à l'utilisation des pectines commerciales.

Ces résultats mettent à la disposition des transformateurs des informations utiles pour la production de jus et de confiture de qualité à partir de bananes plantain surnatures.

\section{Conflit d'intérêt}

Les auteurs déclarent qu'il n'y a aucun conflit d'intérêts pour la publication de l'article.

\section{Contributions des auteurs}

M.A.Y était l'investigateur principal. Il a effectué les travaux de terrain et les tests de laboratoire. E.K.K a conçu le projet et supervisé le travail. O.K.C a contribué à l'analyse sensorielle. E.F.E a contribué à la réalisation des analyses physico-chimiques.

\section{REFERENCES}

Adopo NA, Amafou AG, Kehe MFK, Fofana V. 1998. Les perspectives d'évolution du circuit de distribution de la banane plantain en Côte d'Ivoire. In banana and food security, Proceeding of the International Symposium held in Douala, 537-553

ANADER. 1998. Agence Nationale pour le Développement Rural. Enquète de base, ANADER, 1996-1997.

Anon. 1981 Bibliografiacorta sobre jugos (33 referencia : 1967-79). $4 \mathrm{PP}$

Belitz H-D, Grosch W. 1999. Food Chemistry $\left(2^{\text {nd }}\right.$ edn). Springer - Verlag: Berlin.

Byaruagaba-Bazirake GW, Van Ransburg P, Kyamuhangire W. 2012. Characteristics of enzyme-treated banana juice from three cultivars of tropical and subtropical Africa. African J. Food Sciences Technology, 3(10): 277-290.

Casimir DJ, Jayaraman, KS. 1971. Banana drink, a new canned product. J. Food Research. Q.31: 24-27.

Dupaigne P. 1974. A propos de l'extraction d'un jus de banane en vue de la production de la bière de banane. Fruits, 29: 821 - 822.

Dupaigne P, Dalnic R. 1965. Boisson s nouvelles à base de fruits.I.Bananes. Fruits, 20: 571-575.

Eric. 1990. Mécanismes de gélification des pectines. Systems Bio Industries (SBI), Centre de Développement Alimentaire, Baupte, Carentan ; 33-47.

Embs RJ, Markakis P. 1965. The sulfur dioxide inhibition of enzymatic browning. Paper presented at the $25^{\text {th }}$ annual meeting. Inst. Food Technology, Kansas city, MO.

Ezoua P. 1995. Jus de fruit de rônier (borassus aethiopum Mart). Caractéristiques physico-chimiques et essai de stabilisation pour la conservation. Thèse de doctorat Université de Cocody Abidjan, 105.

Ferrer OJ, Otwell WS, Marshall MR. 1989 Effect of bisulfite on lobstershellphenoloxidase. J. Food Sciences, 54: 478-480.

FAO (Food and Agriculture Organization) 1997. Chemical analysis manual for food and water (5th edn). FAO Rome 1: 20-26. Food and Agriculture Organization (FAO) 1997b. Annual Report on Food Quality Control, 1: 1113.

Galeazzi MAM, Sgarbieri VC. 1981. Substrate specificity and inhibition of polyphenoloxydase (PPO) from à dwarf variety of banana (Musa cavendishii L.). J. Food Sciences, 46 : 1404-1406.

Jehl Bruno, Madet Nicolas. 2004. L'acide ascorbique et son utilisation en tant que additive alimentaire dans les industries 
alimentaire, Université Pari VII Val de Marne.

Koffi E, Sims CA, Bates RP. 1991 Viscosity reduction and prevention of brow ning in the preparation of clarified banana juice. J. Food Quality, 14: 209-218.

Madero CF, Finne G. 1982. Properties of phenoloxidase isolated from gulf shrimp. In Proceedings of the Seventh Annual Tropical and Subtropical Fisheries Technological Conference of the Americas. New Orleans, LA, 328339.

MAO WW. 1974. Banana fruit technology. I. Dehydration of banana puree by drum drying. II. Properties of amylase in banana. J. Food Sciences, 46: 14001403.

MINAGRI, 2004. Ministère de l'Agriculture.

Recensement national agricole 2004, MINAGRI.

Ndabikunze BK, Masambu BN, Tiisekwa BPM, Issa-Zacharia A. 2011. The production of jam from indigenous fruits using baobab (Adansonia digitata L.) powder as a substitute for commercial pectin. African J. Food Sciences, 5(3): 168-175.

N'da Adopo A, Amafon Aguie G, Kehe H, Kamara F, Fofana V. 1998. Les perspectives d'évolution du circuit de distribution de la banane plantain en
Côte d'Ivoire. In: Banana and Food Security. International symposium, Douala, Cameroon, 10-14 november 1998.

Palmer JK. 1971. The banana. In The Biochemistry of Fruits and Their Products (vol 2), Hulme AC (ed). Academic Press, London; 65-105.

Soler A, N'da Adupo A. 1991. Réduction des pertes post-récolte du plantain. Projet PNUD/FAO IVC/87/003. Rapport final. Abidjan, Cote d'Ivoire : CIRADIRFA, 57.

Sreekantiah KR, Jaleel SA, Rao RTN. 1971. Utilization of fungal enzymes in liquefaction of soft fruits and extraction and clarification of fruits juices. J. Food Sciences, 8: 201-203.

Stover RH, Simmonds NW. 1987. Bananas ( $3^{\text {rd }}$ edn). John Wiley and Sons: New York.

UNITED FRUIT CO. 1961. Banana. Ripeninng Manual United Fruit. Sales Corp.: Boston.

Vámos-Vigyázó L. 1981. Polyphenoloxidase and peroxidase in fruits and vegetables. Crit. Rev. Food Sci. Nutr., 15: 49-127.

Viquez F, Lastreto C, Cooke RD. 1981. A study of the production of.Clarified.Banana juice using pectinolitic enzyme. J. Food Technology, 16: 115 - 125. 